\title{
Modelo de Enfermería para optimizar sistemas de salud
} Nursing Model to Optimize Health Systems

\section{Modelo de enfermagem para otimizar os sistemas de saúde}

Doris Grinspun, RN., MSN., PhD., LLD (hon), Dr (hc), O.ONT. *

\begin{abstract}
Resumen
Introducción: Los gobiernos de todo el mundo buscan diferentes estrategias para ampliar el acceso a los servicios de salud, abaratar costos y optimizar resultados. Objetivo: Presentar el contexto socio-político de salud en el que se sitúan las guías de buenas prácticas basadas en la evidencia de la Asociación de Enfermeras de Ontario. Temas de reflexión: La discusión se centra en los desafíos claves de salud y atención sanitaria que enfrentan diversos países del mundo, y las amenazas y oportunidades que éstos presentan para Enfermería. Se enfatiza la necesidad del uso de evidencia como una herramienta central pero no la única para optimizar los resultados de salud de los ciudadanos. Conclusiones: Los cuatro componentes principales que facilitan un modelo de atención efectivo corresponde a: Recursos humanos adecuados; trabajo interprofesional positivo; expansión del rol de las enfermeras profesionales; y la práctica basada en la evidencia. Todos estos componentes son necesarios para alcanzar una atención de acceso universal, oportuna y centrada en la persona. [Grinspun D. Modelo de Enfermería para optimizar sistemas de salud. MedUNAB 2017; 20(2): 224-234].
\end{abstract}

Palabras clave: Enfermería; Mejoramiento de la Calidad; Práctica Clínica Basada en la Evidencia; Guía de Práctica Clínica; Enfermería Basada en la Evidencia.

\begin{abstract}
Introduction: Governments around the world seek different strategies to expand access to health services, lower health costs and optimize health results. Objective: To present the socio-political context in which the best practices guidelines program of the Registered Nurses' Association of Ontario (RNAO) is situated. Reflection Topics: The discussion focuses on the key health and healthcare challenges faced by different countries around the world, and the threats and opportunities they present for Nursing. Emphasis is placed on the need to use evidence as a central tool, but not the only one to optimize the health outcomes of citizens. Conclusions: The four main components that facilitate an effective care model correspond to adequate human resources, positive inter professional work, scope expansion of the role of registered nurses (RNs), and evidence-based practice. All of these components are necessary to achieve universal, timely and person-centered access care. [Grinspun $D$ Nursing Model to Optimize Health Systems. MedUNAB 2017; 20(2): 224-234].
\end{abstract}

Keywords: Nursing; Quality Improvement; EvidenceBased Practice; Practice Guideline; Evidence-Based Nursing.

\footnotetext{
* Registered Nurse, master of Science in Nursing, doctor of Philosophy, doctor of Law (hon), doctor Honouris Causa, gerente general, Asociación de Enfermeras Profesionales, Ontario, Canadá (RNAO)
}

Correspondencia: Doris Grinspun. E-mail: dgrinspun@rnao.ca 


\section{Resumo}

Introdução: Os governos do mundo procuram estratégias diferentes para ampliar o acesso aos serviços de saúde da comunidade, reduzir os custos e otimizar os resultados. Objetivo: Apresentar o contexto de saúde sócio-política, no qual estão localizados os guias de boas práticas, baseados em evidencias, da Associação de Enfermeiras de Ontário. Questões para a reflexão: A discussão centra-se nos principais desafios no cuidado da saúde enfrentados, por diferentes países, em todo o mundo e às ameaças e oportunidades que apresentam para a Enfermagem. A ênfase é colocada na necessidade de usar a evidência como uma ferramenta central, mas não a única para otimizar os

\section{Introducción}

\section{Contexto internacional}

Los gobiernos de todo el mundo buscan modos de ampliar el acceso a los servicios de salud, abaratar costos y optimizar resultados. Estas metas se acentúan frente a una realidad donde las variaciones en la práctica clínica persisten y así también el daño de estas a los ciudadanos (1). Hace más de 30 años Wennberg y Gittelsohn (1973) describieron por primera vez la variación en los patrones de tratamiento en Nueva Inglaterra y otros lugares de los Estados Unidos (2). Desde entonces, los investigadores han seguido documentando grandes variaciones en el uso del servicio y el gasto sanitario en diversas regiones geográficas (3). Estas notables diferencias prácticas en el diagnóstico, tratamiento y manejo de los pacientes continúan impregnando el cuidado de la salud en el mundo entero y así también lo hacen sus problemáticos resultados.

Un elemento importante para reducir la variación en servicios clínicos es la disponibilidad y utilización consistente de la evidencia clínica por profesionales de salud. Las guías de práctica clínica que contienen recomendaciones basadas en la evidencia sirven para este propósito. Diversos grupos ofrecen guías desarrolladas rigurosamente, incluyendo Grupo de Trabajo de Servicios Preventivos (USPSTF), Directriz de Desarrollo Internacional (GIN), y la Asociación de Enfermeras Registradas de Ontario (RNAO). Las guías de práctica clínica son declaraciones que incluyen recomendaciones para la práctica basadas en una revisión sistemática de la evidencia, junto con los beneficios y los daños de las intervenciones destinadas a optimizar la atención y los resultados del paciente (4).

Es en este contexto, el programa de Guías de Buenas Prácticas (BPG) dirigido por la RNAO ha tomado auge internacional. Los BPG de RNAO se basan en la mejor evidencia e incluyen recomendaciones para optimizar resultados clínicos y de salud. Estas guías van dirigidas a enfermeras, otros profesionales de la salud, administradores, educadores y planeadores de políticas de salud. Las Best Practice Guidelines (BPGs) ofrecen una evaluación de la resultados de saúde dos cidadãos. Conclusões: Os quatro principais componentes que facilitam um modelo de atendimento eficaz correspondem a: Recursos humanos adequados; trabalho interprofissional positivo; expansão do papel das enfermeiras profissionais; e à prática baseada em evidências. Todos esses componentes são necessários para alcançar cuidados de acesso universais, oportunos e centrados na pessoa. [Grinspun D. Modelo de enfermagem para otimizar os sistemas de saúde. MedUNAB 2017; 20(2): 224-234].

Palavras chave: Enfermagem; Melhoria de Qualidade; Prática Clínica Baseada em Evidências; Guia de Prática Clínica; Enfermagem Baseada em Evidências.

calidad de la literatura científica relevante y de los beneficios y daños probables de una intervención particular. Estas permiten seleccionar las intervenciones que han demostrado mejores resultados, a ser consideradas para el paciente específico y en función de sus preferencias. El Programa BPG de RNAO es amplio y multifacético, incluyendo desarrollo de guías, apoyo activo para la implementación y un sistema internacional de datos para la evaluación de resultados.

Cabe notar que si bien el uso de evidencia es vital para optimizar resultados, no es suficiente para avanzar a un sistema de salud efectivo y eficiente. De aquí que lo que sigue es un modelo, que si bien no tiene como intención ser exhaustivo, incluye varios de los elementos principales para avanzar hacia la efectividad del sistema de salud.

\section{Modelo para avanzar hacia un sistema de salud efectivo}

\section{¿Qué es un sistema de salud efectivo?}

La Organización Mundial de la Salud en el año 2005, considera que un sistema de salud comprende todas las organizaciones, instituciones, recursos y personas cuya finalidad primordial es mejorar la salud (5). Un sistema de salud necesita personal, fondos, información, suministros, transporte, comunicaciones, orientación y direccionamiento general. También debe prestar servicios, que respondan a las necesidades y sean equitativos desde el punto de vista financiero, al tiempo que se dispense un trato digno a los usuarios.

Las malas condiciones de los sistemas de salud en muchas partes del mundo en vía de desarrollo son uno de los mayores obstáculos al incremento del acceso a la atención sanitaria esencial. Así también hay países adinerados como Estados Unidos con resultados graves de ineficiencia donde gran parte de la población carece de acceso a la atención por causa de disposiciones poco equitativas en materia de protección social y donde los costos son cada vez mayores por causa del uso ineficaz de los recursos (6). 
Cada vez más se reconoce que para mantener y mejorar el grado de salud de la población del mundo los gobiernos deben diseñar sistemas de salud sólidos y eficientes, que presten buenos servicios preventivos y curativos a las mujeres, los hombres y los niños sin distinción alguna (5).

\section{Modelo de políticas de recursos humanos profesionales para avanzar un sistema de salud efectivo}

La base del modelo RNAO es el principio de acceso universal, oportuno, y con una atención centrada en la persona. Sobre este principio se levantan cuatro pilares esenciales para mejorar y optimizar los resultados de salud y lograr la efectividad del sistema de salud (Figura 1). Estos son:

Recursos humanos adecuados: Número de enfermeras, médico y otros profesionales

$\square$ Trabajo interprofesional positivo: Respeto mutuo y colaboración auténtica entre todos los participantes del equipo de salud, y especialmente entre médicos y enfermeras

$\square$ Expansión del rol de las enfermeras profesionales: Conocimiento amplio y capacidad de ejercer todas las competencia clínicas adquiridas

Práctica basada en la evidencia: Integración de la experiencia clínica individual con la mejor evidencia clínica externa disponible de la investigación sistemática y los valores del paciente (7).

\section{EFICACIA DEL SISTEMA DE SALUD}

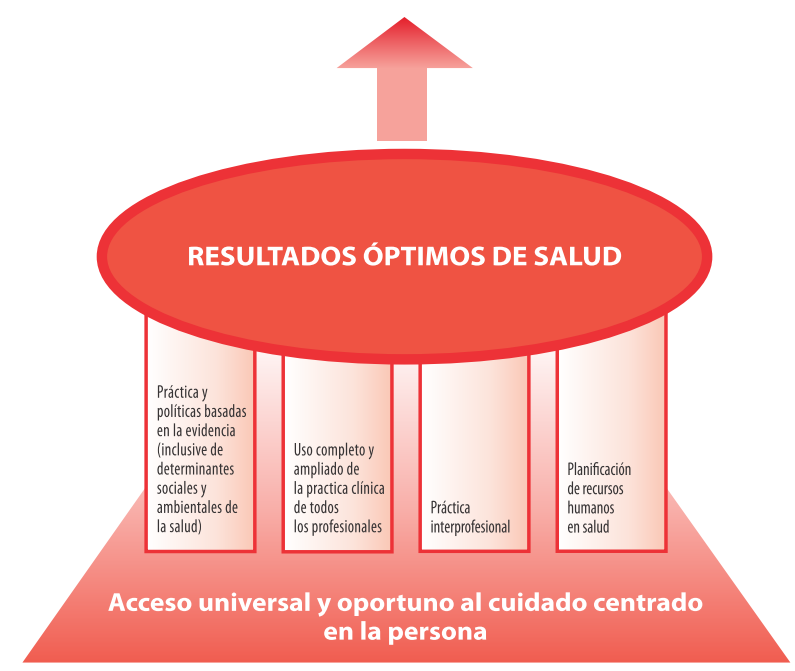

Fuente: RNAO, 2016*

* Registered Nurses' Association of Ontario (RNAO). (2016). Mind the Safety Gap in Health System Transformation: Reclaiming the Role of the $R N$. Retrieved from http://rnao.ca/ sites/rnao-ca/files/HR_REPORT_May11.pdf

Figura 1. Eficacia del sistema de salud.
Cada uno de estos cuatro pilares es de gran importancia y su transcendencia ha sido visualizada en resultados de investigación.

\section{Primer pilar: Recursos humanos adecuados}

Una revisión de 70 años de investigación sobre la eficacia de la enfermera profesional realizada en el año 2017, muestra que la vinculación de enfermeras dan mejores resultados clínicos y financieros. Enfermera profesional es entendida en Canadá y Estados Unidos como la Registered Nurse o $\mathrm{RN}$, y en Latinoamérica como la enfermera universitaria (8).

De los 626 estudios de investigación identificados por la revisión de RNAO, más del 95\% mostró que las enfermeras profesionales tienen un impacto positivo, mayor que las enfermeras técnicas o auxiliares en una amplia variedad de resultados de salud a pacientes, organizaciones sanitarias y sistemas de salud. Estos incluyen la reducción de la mortalidad, reducción de la morbilidad (por ejemplo: caídas, úlceras de presión, infecciones, etc), el aumento de la calidad de la atención, el aumento de la satisfacción de los pacientes, aumento en la seguridad organizacional y disminución en los costos (Figura 2 y 3). Esta base de datos es la más amplia y extensa que existe de forma pública y se puede acceder en la página web www.RNAO.ca(9).

\section{Segundo pilar: cuidado interprofesionalpositivo}

El cuidado interprofesional no es algo nuevo. Se ha dado por décadas basado en equipos en general liderados por un médico. La diferencia está en que durante la última década se dan innovaciones en la forma como estos equipos interprofesionales se desenvuelven, especialmente en la ruptura con modelos anteriormente jerárquicos y la inserción del paciente y su familia como parte central del equipo. Se piensa que estas nuevas formas de desempeñarse como equipo fortalecen la atención al paciente y los sistemas de salud. La discusión en la literatura presenta la complejidad y esfuerzos deliberados a niveles organizacionales que este cambio requiere (10).

La RNAO define el cuidado interprofesional como: Múltiples disciplinas de salud con diversos conocimientos y habilidades que comparten un conjunto integrado de metas y que utilizan la colaboración interdependiente que implica la comunicación, el intercambio de conocimientos y la coordinación de servicios para proporcionar servicios a pacientes/clientes y sus sistemas de cuidado (10).

Tan importantes son los cuidados interprofesionales positivos que la RNAO ha dedicado a este tema una guía completa basada en la evidencia, titulada "Desarrollo y mantenimiento de la atención de salud interprofesional: La optimización de los pacientes/clientes, la organización y los resultados del sistema" (10). Su propósito es apoyar con recomendaciones basadas en la evidencia, el desarrollo y mantenimiento de la atención de equipos de salud 


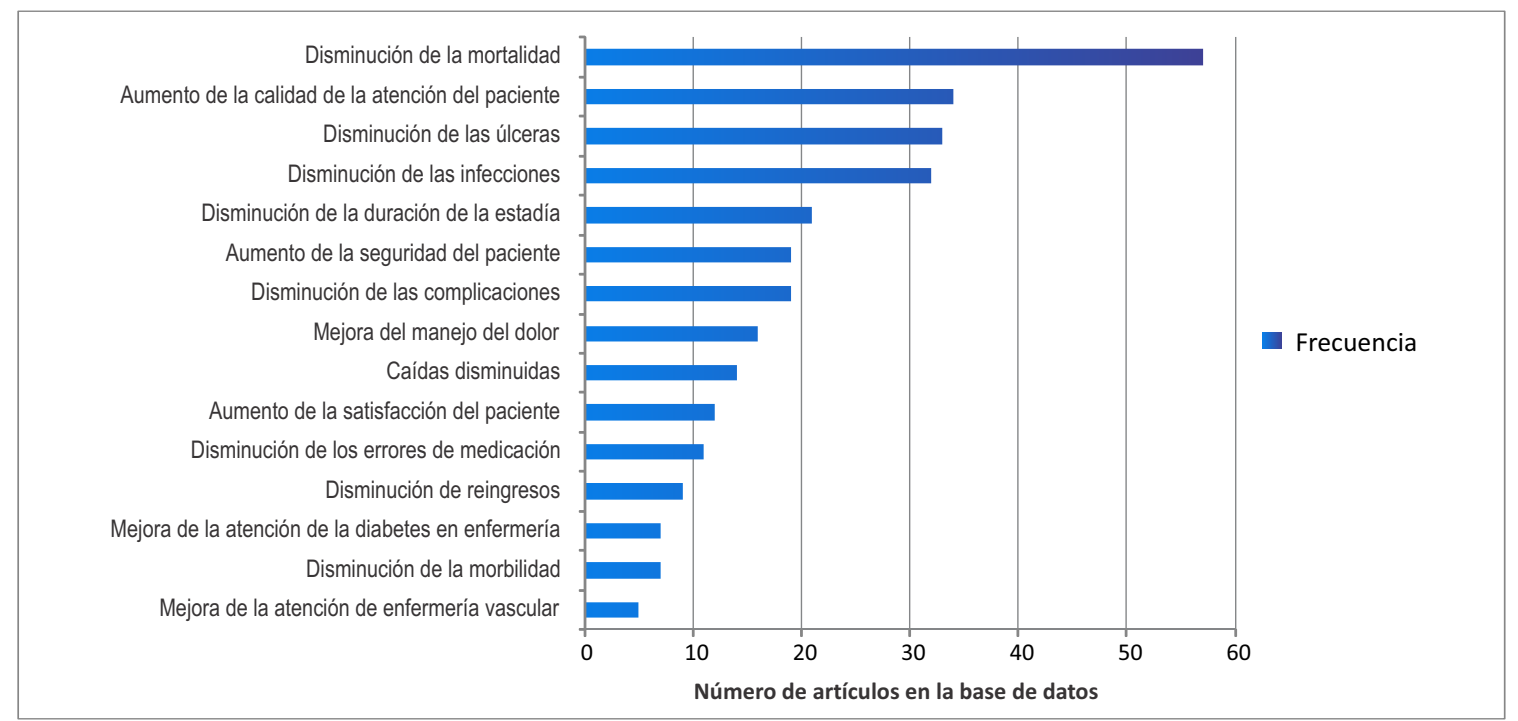

Fuente: Tomado de Registered Nurses' Association of Ontario (RNAO). RN Effectiveness Database.

Figura 2. Resultados de salud beneficiosos para pacientes.

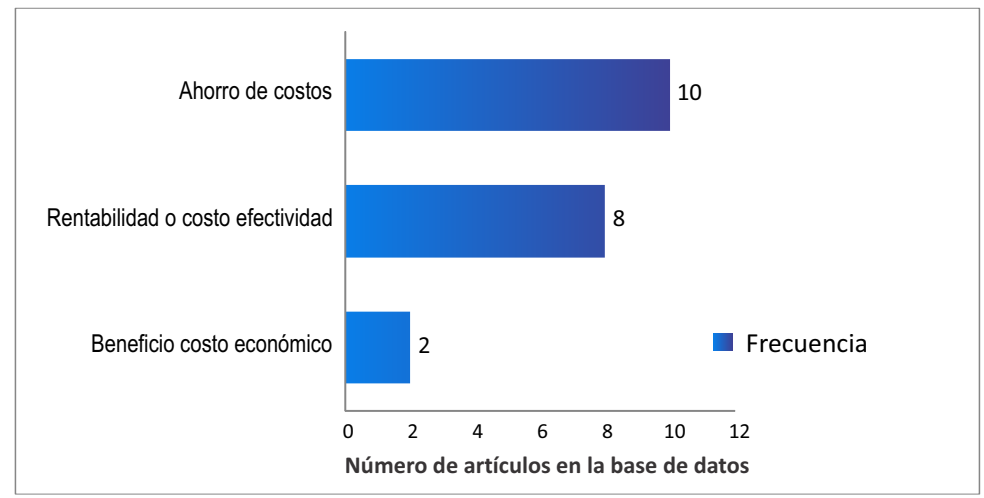

Fuente: Tomado de Registered Nurses' Association of Ontario (RNAO). RN Effectiveness Database.

Figura 3. Resultados financieros positivos.

interprofesional para conjuntamente optimizar la atención a pacientes y sus resultados. Dicha guía está dedicada a enfermeras y otros profesionales de la salud en todos los roles y prácticas, administradores en todos los niveles de una institución prestadora de servicios de salud, estudiantes; educadores; investigadores, gobiernos; organizaciones profesionales, grupos laborales; y los organismos normativos federales, provinciales y territoriales.

La guía comienza delineando nueve principios (10):

1. Los equipos más eficaces producen mejores resultados.

2. Los equipos de colaboración son más eficaces que los proveedores individuales de servicios de salud.

3. Los pacientes/clientes forman parte integrante de los equipos interprofesionales.

4. La experiencia total de los miembros del equipo es mayor que la suma de sus partes y produce mejores resultados.

5. Los servicios son holísticos y coordinados en todo el espectro de proveedores.
6. La recompensa de los mejores resultados del paciente/cliente es el mejor incentivo para los equipos interprofesionales de alto funcionamiento.

7. Hay poder arraigado y diferenciales de estatus que son discutidos por el equipo para apoyar el equipo eficaz.

8. Es necesario reconocer la diferencia de poder entre los proveedores de atención de salud y entre pacientes y clientes. Es necesario que se abordan mediante políticas organizacionales.

9. Los marcos financieros e incentivos fomentan los servicios de salud interprofesionales basados en trabajo en equipo.

En esta guía, la atención interprofesional ejemplar en un ambiente de trabajo saludable, es un producto de la sinergia entre los equipos de salud que demuestran experiencia en seis dominios claves que son (10):

1. Experiencia en el cuidado 
2. Poder compartido

3. Liderazgo colaborativo

4. Optimizar la profesión y el rol que cada uno cumple

5. Toma de decisiones compartida

6. Funcionamiento efectivo del grupo.

Esos seis dominios son fundamentales para transformar los ambientes de trabajo en un espacio interprofesional colaborativo, mientras que los componentes fundamentales apoyan e influyen en cada dominio para lograr la atención interprofesional ejemplar para pacientes y sus redes de apoyo. Cuando la atención interprofesional ha sido implementada y sostenida con éxito, la mejora de calidad continúa y seguridad al paciente se producen en tres niveles. Micro, para pacientes y para proveedores interprofesionales; meso, para la organización donde estos pacientes reciben atención de salud (por ejemplo: hospital, hogar de ancianos, etc); y macro, y el sistema de salud.

\section{Tercer pilar: expansión del rol de las enfermeras profesionales}

Este pilar representa la necesidad que las enfermeras profesionales tengan conocimientos amplios y la capacidad de ejercer todas sus competencias clínicas. Muchos son los casos de enfermeras profesionales que se ven bloqueadas de ejercer todas sus competencias por lugares laborales restrictivos. La efectividad de los sistemas de salud y el acceso oportuno de usuarios pueden mejorar significativamente si todos los profesionales de la salud están ejerciendo todas sus competencias clínicas y trabajando en equipos interprofesionales de alto funcionamiento $(10,11)$, que usan evidencia para otorgar una atención clínica apropiada, segura y de calidad (12).

La expansión del rol de la enfermera puede incluir prescripción de algunos exámenes de laboratorio para detectar, por ejemplo, infecciones urinarias; diagnóstico de falencias comunes como es otitis media; y prescripción de medicamentos. Este tipo de expansión se está dando hace 15 años en Gran Bretaña (13-19) y varias provincias de Canadá (16).

\section{Cuarto pilar: práctica clínica basada en la evidencia}

Si bien es cierto que los tres pilares anteriores son indispensables para avanzar un sistema sanitario eficiente, estos no lograrán resultados eficaces óptimos si no van acompañados de una práctica clínica basada en la evidencia. Es por esto que la RNAO creó el programa de guías clínicas y de entorno laboral basados en la evidencia. El programa fue lanzado en el año 1999 por la RNAO en asociación con el Ministerio de Salud de la provincia de Ontario. Esta es la más grande del país y cuenta con $13,982.984$ personas, lo que representa 38.5 de la población de Canadá(17).

El propósito del programa desde su inicio ha sido apoyar a enfermeras otorgándoles guías de mejores prácticas basadas en evidencia para el cuidado de pacientes en todos los ámbitos de salud. Las metas del programa son:

- Mejorar la consistencia y calidad de la atención de enfermería

- Aumentar el acceso a servicios de enfermería de calidad

- Difundir los recursos lo más ampliamente posible para que se obtengan los máximos beneficios para los pacientes, las enfermeras y el sistema de atención de la salud.

Los objetivos del programa son:

- Desarrollar BPGs

- Actualizar los BPGs cada cinco años

- Difundir y apoyar activamente la implementación de BPGs a través de mecanismos eficaces y usando ciencia de implementación

- Evaluar los procesos de desarrollo, difusión, implementación y resultados asociados con los BPGs

\section{Desarrollo de las Guías de Buenas Prácticas RNAO}

Las Guías RNAO son desarrolladas sistemáticamente. Contienen declaraciones basadas en la evidencia y recomendaciones para enfermeras y el equipo clínico interprofesional, entornos laborales, y educadores tanto dentro de sistemas sanitarios como académicos. Las guías ofrecen una evaluación de la calidad de la literatura científica pertinente y una evaluación de los beneficios y daños probables de una intervención en particular (Figura 4). Las BPG son utilizadas como una herramienta para mejorar la toma de decisiones y apoyar la provisión de la mejor atención posible basada en la evidencia. La Figura 5 muestra gráficamente los tipos de guías que RNAO ofrece. La Figura 6 muestra los tipos de recomendaciones incluidas en los BPG clínicos de RNAO. Estas recomendaciones basadas en la evidencia influyen en la práctica, a nivel individual y de equipo, de políticas de salud y educación para asegurar la adopción de BPG y el uso sostenido (18).

\section{Proceso de desarrollo de la guía RNAO}

La RNAO tiene dos décadas de experiencia en el desarrollo de guías basadas en la evidencia. El proceso que usa incluye siete pasos y estos son refinados continuamente cumpliendo los estándares internacionales más altos, y liderando con metodologías fácil de usar, eficaces y eficientes. Los siete pasos incluyen la selección de temas, el uso de un panel de expertos, la revisión sistemática, la formulación de recomendaciones, la revisión de las partes interesadas, la publicación y la actualización de la guías cada 5 años. Cada uno de estos pasos están descritos dentro de cada una de las guías como también en su página web: www.RNAO.ORG.ca/BPG(9).

Durante el 2017 RNAO comenzó su alineación de nuevas guías y nuevas ediciones de guías existentes con los marcos 


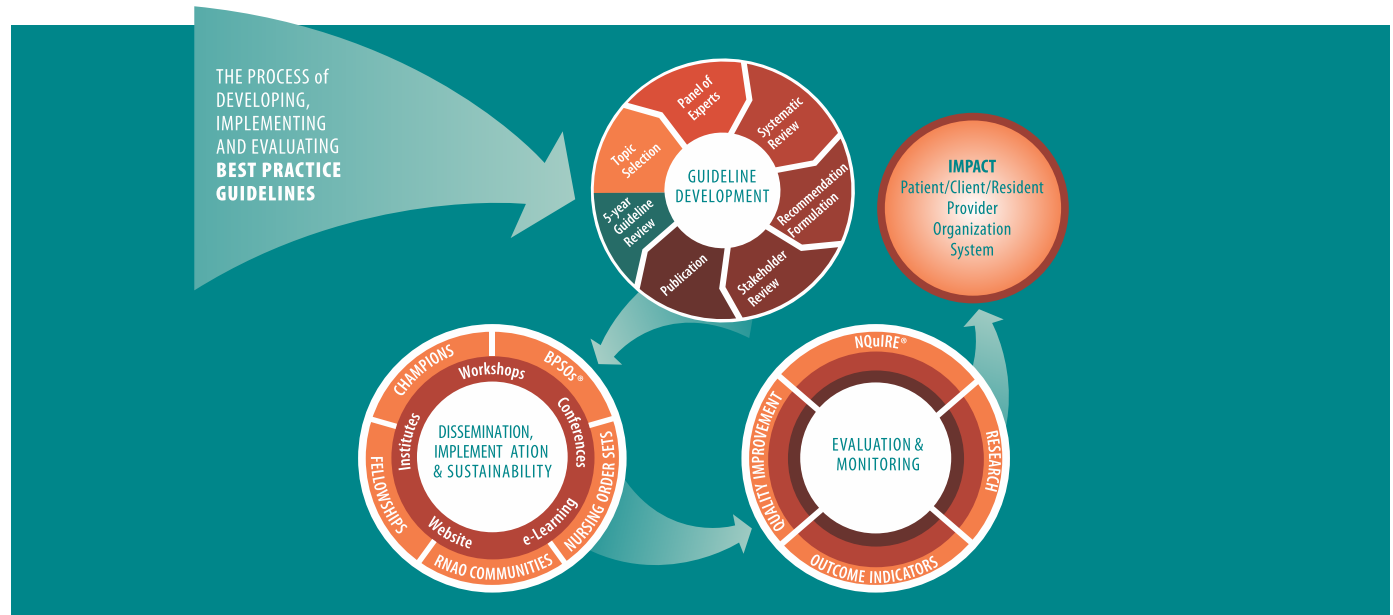

Fuente: RNAO, Registered Nurses'Association of Ontario (RNAO).

* Registered Nurses'Association of Onario (RNAO) (n.d.). Programa de Directrices sobre Mejores Prácticas de la RNAO.

Retrieved from http://rnao.ca/sites/rnao-ca/files/iaBPG_brochure_Spanish.pdf

Figura 4. Modelo RNAO: Desarrollo, Implementación y Evaluación de Guías de Buenas Prácticas.

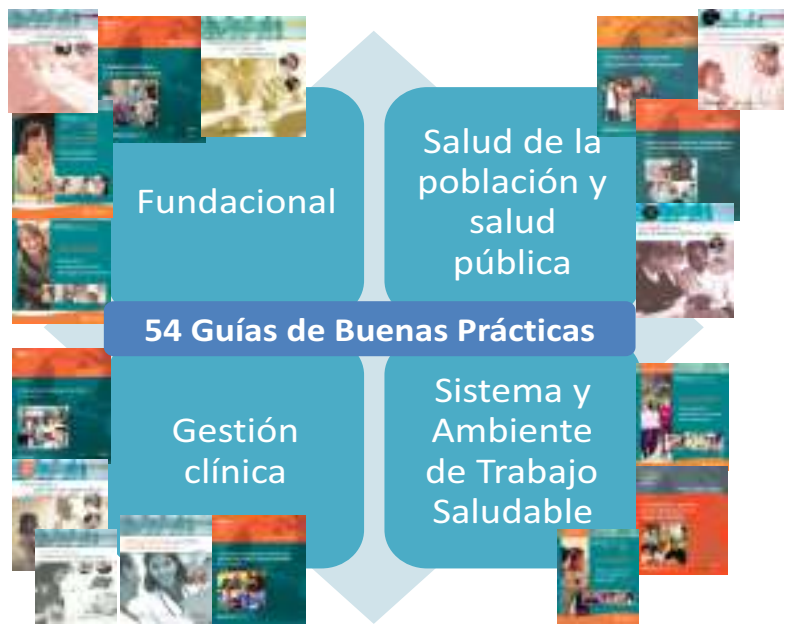

Fuente: Tomado de Registered Nurses' Association of Ontario (RNAO)(C.

Figura 5. Clasificación de las Guías de buenas prácticas RNAO.
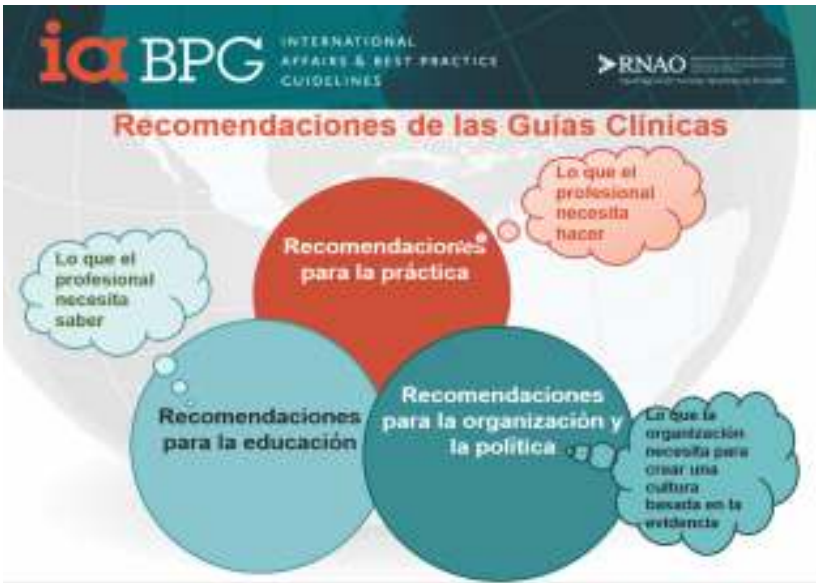

Fuente:Tipos de recomendaciones de Guías de buenas prácticas RNAO

Figura 6. Clasificación de las Guías de buenas prácticas RNAO. 
GRADE y GRADE CERQual empleados para categorizar la fuerza de una recomendación. Esta se basa en la calidad de la evidencia, el grado de incertidumbre sobre el equilibrio entre los efectos deseables e indeseables, el grado de incertidumbre o variabilidad de los valores y preferencias y el grado de incertidumbre sobre si la intervención representa un uso racional de los recursos. Con esta metodología de desarrollo de la guía RNAO se dan cambios importantes al proceso de desarrollo du sus guías, ya que antes de la revisión sistemática, el equipo de desarrollo ahora lleva a cabo un análisis de brechas para determinar áreas específicas de enfoque para el propósito, el alcance, las preguntas de investigación y los resultados de la guía. Las conclusiones de este proceso informan al grupo de expertos, el cual finaliza la orientación de la guía y preguntas a responder.

\section{Implementación de las Guías de Buenas Prácticas RNAO}

RNAO ha usado desde el comienzo del programa enfoques de implementación múltiples a nivel individual, organizacional y de sistema. El más importante a nivel individual es el entrenamiento de "champions" o en castellano "campeones". Los Campeones de BPGs son enfermeras y otras personas a quienes les apasiona mejorar la práctica de la enfermería y la atención a pacientes en su organización. Los campeones influencian el uso de evidencia a nivel de la unidad de servicio y la organización. Esto lo hacen modelando directamente una práctica clínica basada en la evidencia, como también asumiendo diversos roles, tales como concienciar sobre las mejores prácticas para su organización, influenciar a los grupos y comités para considerar estas mejores prácticas, movilizar, coordinar y facilitar la capacitación y el desarrollo de personal profesional en la implementación de las mejores prácticas (20). Ellos son centrales para cerrar la brecha que en general existe entre la evidencia y la práctica apoyando una calidad continua y así avanzando la seguridad al paciente y mejores resultados clínicos y de salud (21).

El más importante foco de implementación a nivel organizacional son los Best Practice Spothlight Organizations or BPSOs $(22,23)$. Esta es una designación formal que se obtiene a través de un convenio de tres años con RNAO. El objetivo de los BPSOs es influir en la adopción de guías de buenas prácticas para avanzar los mejores resultados a pacientes, organizaciones y sistemas de salud. Hay dos tipos de BPSOs y dos categorías. Los dos tipos son BPSO Servicios que se enfocan en implementar las guías RNAO en las instituciones que prestan servicios clínicos como ser atención primaria, domiciliaria, hospitalaria, hogares de ancianos, etc. $(24,25)$; y los BPSOs Académicos que se enfocan en la integración de las guías RNAO en la malla curricular para impactar el aprendizaje del estudiante (21). Las dos categorías de BPSOs son el BPSO anfitrión (host en Ingles) que desarrollan un contrato con la RNAO para supervisar la BPSO Designación en su jurisdicción. Los
BPSO anfitriones trabajan con RNAO, liderando BPSOs de servicio o académicos en su jurisdicción para aplicar y trabajar para convertirse en BPSOs Directos. Los últimos BPSO Directo- firman sus convenios con BPSOs anfitriones para implementar 3 guías clínicas durante un período de 3 años para obtener la designación BPSO. El convenio delinea pautas claras a cumplir y el apoyo que será recibido de su BPSO anfitrión. El programa es sin ánimo de lucro. Otro beneficio importante del programa BPSO es el uso gratuito de las órdenes de enfermería (22).

El programa BPSO tiene también implementación a nivel de todo el sistema de salud que se basan en la aplicación de BPG RNAO como son el proyecto de tabaquismo y dejar de fumar; el programa de buenas prácticas en la atención al adulto en hogares de ancianos; salud mental y adicciones, $\mathrm{u}$ otros. Por motivos de espacio estos y otros no son discutidos en este artículo.

\section{Alcance de las Guías de Buenas Prácticas RNAO}

El alcance de las guías RNAO es impresionante $(17,26)$. En diversos congresos internacionales como son los del Consejo Internacional de Enfermeras (CIE) o Sigma Theta Tau Internacional (STTI) son muchas las enfermeras que presentan los avances sobre el uso de las guías RNAO en sus instituciones. Hay quienes han adoptado estas guías de modo independiente, y otros que lo han hecho a través del convenio BPSO. Para quienes dirigimos el programa, lo más importante es el uso sistematizado a través del convenio BPSO en el cual participan formalmente más de 550 instituciones de prestación de servicios de salud. Entre ellos uno de los más importantes es la Unidad de Investigación en Cuidados de salud (Investén-isciii) en el Instituto de Salud Carlos III los cuales fueron los primeros integrantes del modelo BPSO Host de RNAO (24). Si bien los portones al mundo internacional se abrieron solo en el año 2011, su expansión a través del modelo de BPSOs (Figura 7).

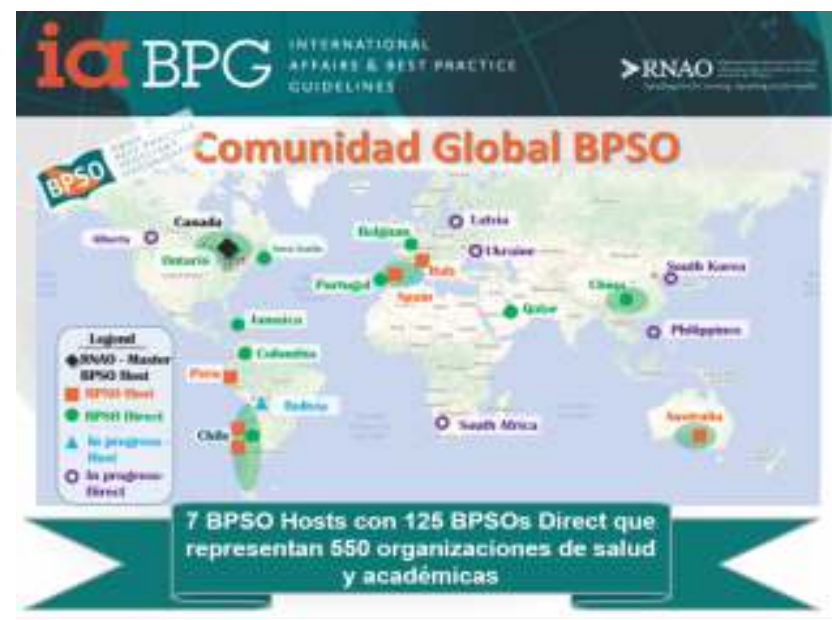

Fuente: Tomado de Registered Nurses' Association of Ontario (RNAO)C.

Figura 7. Alcance de las Guías de Buenas Prácticas RNAO. 


\section{Nursing Quality Indicators for reporting and Evaluation Data-System (NQuIRE): Evaluación de las Guías RNAO}

Nquire fue lanzado en el año 2012 como un sistema de datos de indicadores de calidad derivados de las recomendaciones de práctica dentro de las guías clínicas de RNAO. A través de este sistema RNAO recopila, analiza y reporta a sus BPSOs datos comparativos (trimestral) sobre los indicadores que reflejan la estructura, proceso y resultados de la atención que surgen de la implementación de guías (25).

Usando los resultados de NQuIRE el programa provee ahora a sus BPSOs con impulsores de evidencia. Los impulsores de evidencia son infografías que demuestran el impacto de la implementación de la guía en los resultados clínicos. Los impulsores de evidencia proporcionan datos sobre los resultados en pacientes de diversas guías RNAO. Estos incluyen resultados en personas que residen en hogares de ancianos, por ejemplo en un menor uso de sujetadores; disminución dramática en las lesiones por presión adquiridas en el hospital; menores tasas de incidencia de úlceras por presión y de caídas en atención domiciliaria (BPSO) tanto a nivel local como en el extranjero. Detalles sobre impulsores de evidencia se encuentran en el ApéndiceA.
Muy importante también son los resultados de investigación llevados adelante por diversos BPSOs $(19,20)$.

\section{Conclusiones}

En este artículo se presenta el contexto socio-político de salud en el que se sitúan las guías RNAO. Incluidos en la discusión están un modelo de políticas de recursos humanos profesionales para avanzar un sistema de salud efectivo. Los cuatro componentes principales de este son detallados: Recursos humanos adecuados; trabajo interprofesional positivo; expansión del rol de las enfermeras profesionales; y la práctica basada en la evidencia expresando que todos son necesarios para alcanzar una atención de acceso universal, a tiempo oportuno y centrada en la persona. El artículo luego se vierte en el programa de guías RNAO como una fuente importante para lograr una práctica clínica y de entornos laborales basada en la evidencia. Aquí se comparten las tres fases de desarrollo, implementación y evaluación de las guías son compartidas. El aporte termina con los resultados alcanzados tanto en su adopción en más de 550 instituciones de salud y académicas en diversos países del mundo, como lo más importante que son el impacto en resultados clínicos y de salud para pacientes.

Apéndice A. Ejemplo de Promotores de Evidencia de RNAO publicados en el periodo 2017

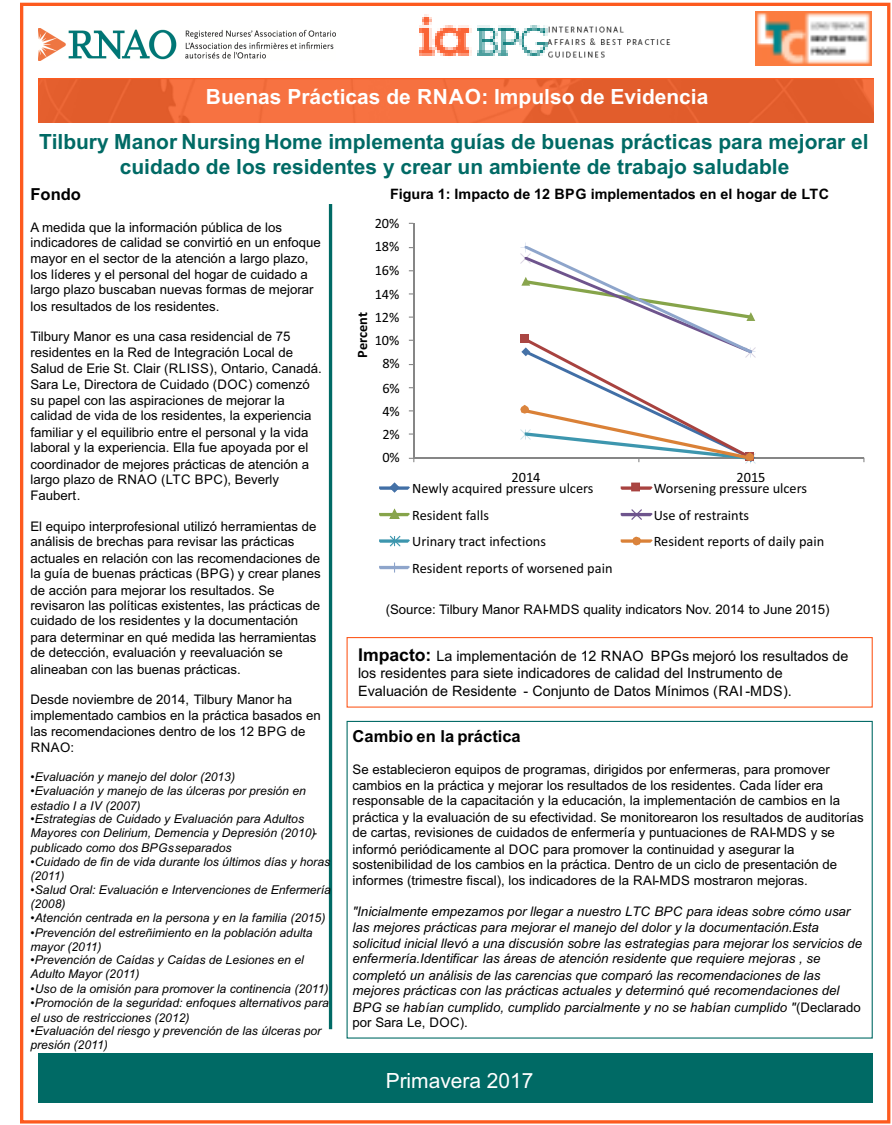




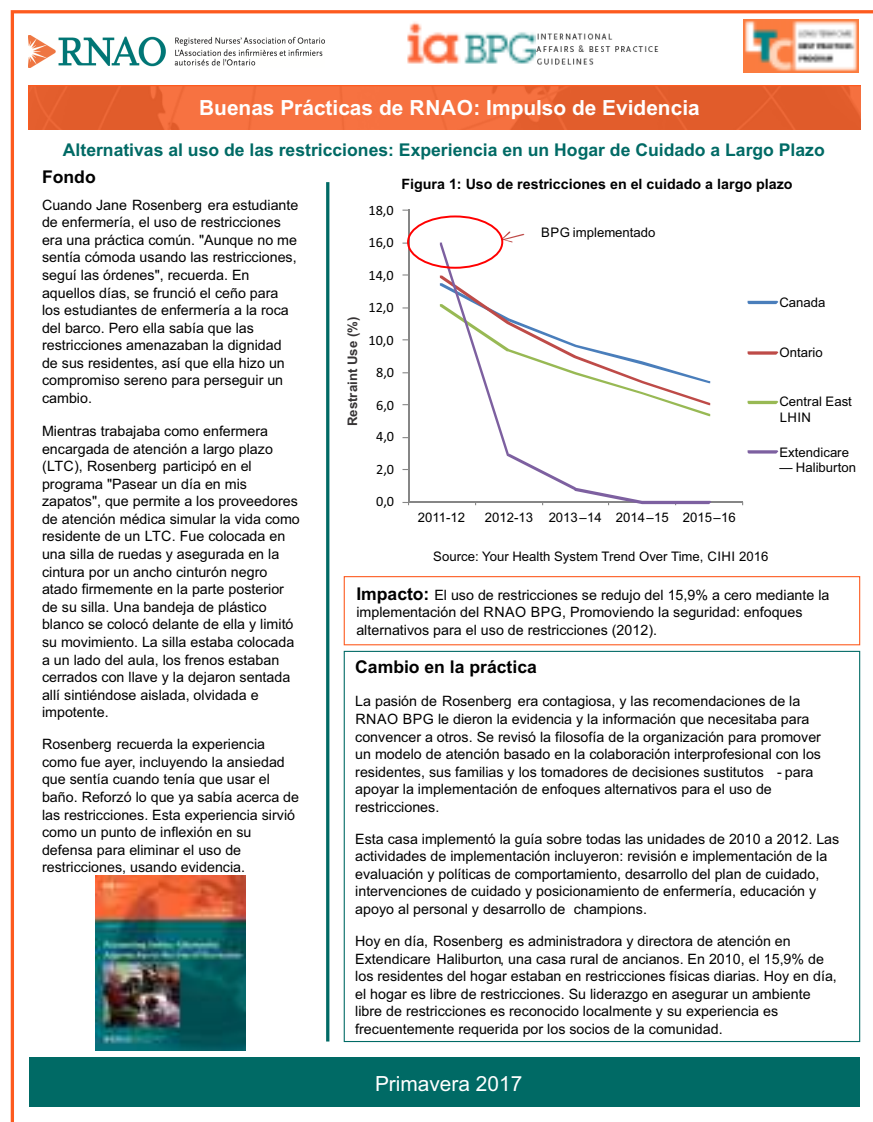

\section{- RNAO}

\section{Buenas Prácticas de RNAO: Impulso de Evidencia}

Implementación de la Guía de Buenas Prácticas para Reducir la Tasa de Incidencia

Evaluación de riesgos y

prevención de las úlceras de

presión

Esta guia ayuda a las enfermeras que
trabajan en diversos entornos de práctica

trabajan en diversos entornos de práctica
para identificar a los adultos que están en

riesgo de úlceras de presión (la terminologí
actual utilizada es las lesiones por presion)

La dirección se proporciona a las

intermeras en la definición de
intervenciones tempranas para la prevención

de lesiones por presión y para controlar las
lesiones de presión de la Etapa I.
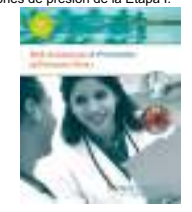

Las lesiones por presión pueden tener un

impacto importante en la calidad de vida y
estado de salud de una persona. Aunque la

prevalencia de lesiones por presión oscila
entre el 0,4 y el 14,1 por ciento en Canadá,

existe una fuerte indicación de que las

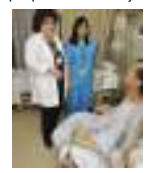

El costo económico de tratar una sola lesión

El costo economico de tratar una sola
por presión oscila entre CAD $\$ 26,800$ a
231,000 [en 2017] y el tratamiento de

lesiones por presión puede aumentar las

$50 \%{ }^{2}$. En Canadáá un mes de atención en la
comunidad por una lesión por presión cuesta

de Lesiones por Presión

Objetivo: Examinar los cambios en los resultados de salud asociados
con la implementación de la guia de buenas prácticas de la RNAO (BPG). Evaluación de Riesgo y Prevención de Uúlceras de Presión (2011), en dos

Medida: Indice de incidencia de lesiones por presión de 2013 a 2010 basado en el sistema de datos NQuIRER.

Mejoramiento Clínico: Disminución del número de pacientes que desarrollaron una o más lesiones de presión nuevas de estadios II a IV
después de la admisión a BPSO hospitalarias, como porcentaje del númer después de la admisión a BPSO hospitalarias, como porcentaje del número de pacientes evaluados con lesiones por presión.

Figura 1: Promedio trimestral de la tasa de incidencia de lesiones po

Inicio de la implementación del BPG

$=0$

is

$5=0$

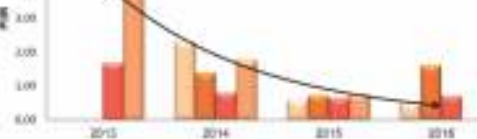

Impacto: La tasa de incidencia de lesiones por presión disminuyó en un $86 \%$ (5.1 a 0.7) de 2013 a 2016 en el hospital internacional BPSO -1 (vea

Cambios en la práctica

Este hospital BPSO-I implementó la guia entre 2012 y 2015 . Las actividades de
implementación incluyeron: politicas y procedimientos alineados con las implementación incluyeron: politicas y procedimientos alineados con las
recomendaciones de la guia; orientación estandarizada y talleres para el person

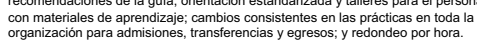
Desde la implementación de esta guia, BPSO-I ha mantenido las actividades de CAD $\$ 9,000^{3}$ 


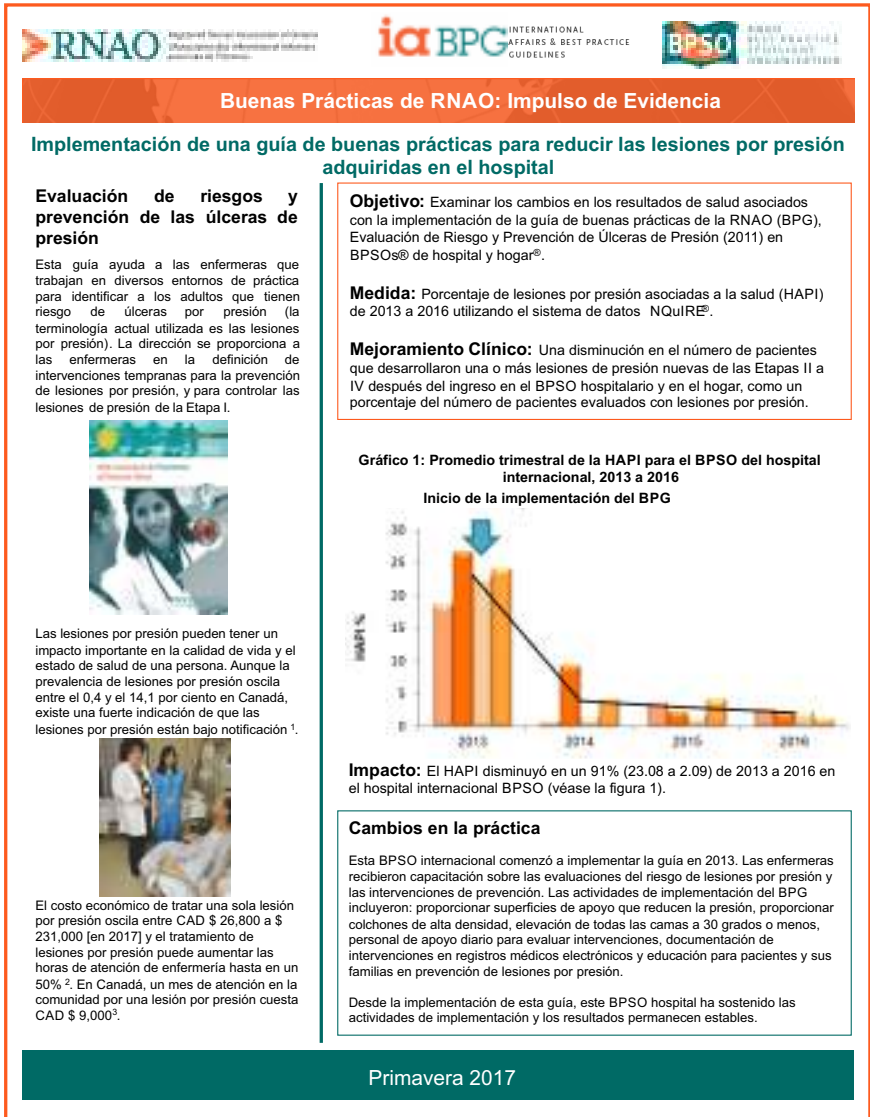

\section{RNAO \\ Buenas Prácticas de RNAO: Impulso de Evidencia}

Implementación de la guía de buenas prácticas para reducir las caídas en adultos mayores

Prevención de caídas y lesiones relacionadas con la caída en el adulto mayor

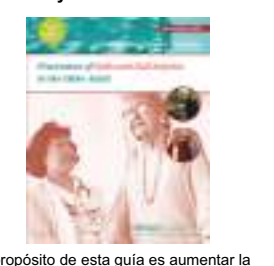

El propósito de esta guia es aumentar habilidades $y$ las habilidades de todos los enfermeros en la identificación de adultos mayores en centros de salud en riesgo de caidas y definin in
prevenir la caida.

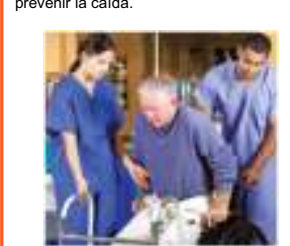

Las caidas son un problema importante de salud
en los adultos mayores, causando lesiones fisicas y los impactos psicológicos que resultan en hospitalizaciones. En Canadá, el $95 \%$ de
fracturas de cadera y el $85 \%$ de las hospitalizaciones relacionadas con lesiones en que los costos directos anuales de atención de la salud son de 2.000 millones de dólares canadienses debido a caidas, y los adultos
mayores representan casi la mitad de estos mayores
costos $^{2}$.

etivo: Para examinar los cambios en los resultados de salud asociados con la implementación de la guía de buenas prácticas de la
RNAO (BPG) Prevención de caílas y lesiones relacionadas con la caida en el adulto mayor (2011), en dos BPSOs ${ }^{\oplus}$ hospitalarios.

Medida: Tasa de caidas por cada 1.000 dias -paciente de 2012 a 2016 thilizando el sistema de datos NQuIRED.

Mejoramiento Clínico: Una disminución en el número de caídas en
BPSOs hospitalarias, como una tasa del número de dias de pacientes.

Figura 1: Tasa promedio de caidas en el hospital de Ontario BPSO I

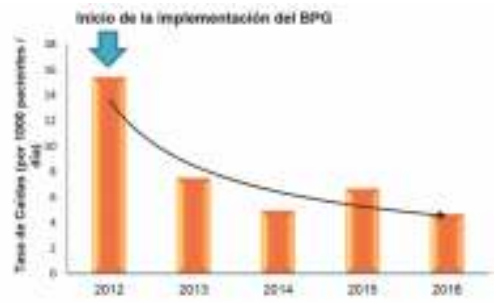

Impacto: La tasa de caídas disminuyó en un 70\% (15,4 a 4,7) de 2013 a 2016 en el BPSO-I del hospital de Ontario (ver Figura 1).

Cambios en la práctica

Entre el 2012 y el 2015, este hospital de Ontario BPSOl implementó completamente la guia de RNAO en toda la organización. Las actividades de implementación incluyeron: capacitación para el personal, educación del paciente,
detección de caidas, una herramienta de documentación posterior a la caida, redondeo deliberado y carteles a la cabecera con los requisitos de transferencia de paciente. Este BPSO compró las camas bajas según lo recomendado y usó
etiquetas engomadas de las caidas para indicar a pacientes en riesgo de caidas, etiquetas engomadas de las caídas para indicara a pacientes en riesgo de caidas. Se
desarrolló una politica de toda la organización para guiar la prevención de caídas y desarrollo una polits
lesiones por caidas.

Desde 2015, esta BPSO ha mantenido las actividades de implementación y los

Primavera 2017 


\section{Agradecimientos}

Al equipo RNAO por su increíble trabajo en el desarrollo de guías de buenas prácticas basadas en la evidencia, a los Promotores de Evidencia que sirven para visibilizar la contribución de Enfermería a los resultados clínicos de pacientes. Así también, aplaudo el compromiso y liderazgo de los BPSOs.

\section{Referencias}

1. Melnyk BM, Grossman DC, Chou R, Mabry I, Nicholson W, DeWitt TG, et al. USPSTF Perspective on EvidenceBased Preventive Recommendations for Children. Pediatrics. 2012; 130(2): e399-e407. Disponible en: https://doi.org/10.1542/peds.2011-2087

2. Wennberg J, Gittelsohn A. Small area variations in health care delivery. Science. 1973; 182(4117):1102-1108. Disponible en: https://doi.org/10.1126/science. 182.4117.1102

3. Newhouse JP, Garber AM, Graham RP, McCoy MA, Mancher M, Kibria A. Variation in Health Care Spending: Target Decision Making, Not Geography. Washington, DC: The National Academies Press; 2013

4. Melnyk BM, Fineout-OverholtE. Evidence-Based Practice in Nursing \& Healthcare. 3a ed. Philadelphia: Lippincott, Williams \& Wilkins; 2014.

5. Organización Mundial de la Salud (OMS). ¿Qué es un sistema de salud? [Internet]. 2005. [citado enero de 2017]. Disponible en: http://www.who.int/features/qa/ 28/es/

6. Du L, Lu W. U.S Health-Care System Ranks as One of the Least-Efficient. [Internet]. 2016. [citado enero de 2017]. Disponible en: https://www.bloomberg.com/news/ articles/2016-09-29/u-s-health-care-system-ranks-asone-of-the-least-efficient

7. Sackett DL, Rosenberg W, Muir-Gray JA, Haynes R, Richardson WS. Evidence based medicine: what it is and what it isn't. BMJ. 1996; 312:71-72. Disponible en: https://doi.org/10.1136/bmj.312.7023.71

8. Registered Nurses' Association of Ontario (RNAO). RN Effectiveness Database. [Internet]. 2017. [citado enero de 2017]. Disponible en: http://rnao.ca/bpg/initiatives/ RNEffectiveness

9. Registered Nurses' Association of Ontario (RNAO). Publications \& Resources. [Internet]. 2017. [citado enero de 2017]. Disponible en: www.rna.org.ca/BPG.

10. Registered Nurses' Association of Ontario (RNAO). Developing and Sustaining Interprofessional Health Care: Optimizing patient, organizational, and system outcomes. [Internet] 2013. [citado enero de 2017]. Disponible en: http://rnao.ca/sites/rnao-ca/files/ DevelopingAndSustainingBPG.pdf

11. Coffey S, Anyinam C. Interprofessional Health Care Practice. 1st Ed. Toronto: Pearson Education; 2014.

12. Grinspun D. Transformando la Enfermería a través de la evidencia. Revista MedUNAB. 2015; 17(3):133-134.

13. Cooper R, Guillaume L, Avery T, Anderson C, Bissell P, Hutchinson A, et al. Nonmedical prescribing in the United Kingdom: developments and stakeholder interests.
Journal of Ambulatory Care Management. 2008; 31(3):244-252. Disponible en: https://doi.org/ 10.1097/01.JAC.0000324670.91153.b4

14. Day CA, Islam MM, White A, Reid SE, Hayes S, Haber PS. Development of a nurse-led primary healthcare service for injecting drug users in inner-city Sydney. Australian Journal of Primary Health. 2011; 17(1):10-5. Disponible en: https://doi.org/10.1071/PY10064.

15. Durán LR. Norma Oficial Mexicana NOM-019-SSA32013, para la práctica de enfermería en el Sistema Nacional de Salud. Revista CONAMED. 2013; 18(1):S34-S41.

16. Registered Nurses' Association of Ontario (RNAO). Registered Nurse Prescribing Referral. [Internet]. 2016. [citado enero de 2017]. Disponible en: http://rnao.ca/ sites/rnao-ca/files/RNAO_RN_Prescribing_HPRAC_Jan_15_2016_Submission-Final.pdf.

17. Ontario Ministry of Finance. Ontario Fact Sheet 2017 Sept. [Internet]. 2017. [citado enero de 2017]. Disponible en: https://www.fin.gov.on.ca/en/economy/ ecupdates/factsheet.html.

18. Bajnok I, Grinspun D, Lloyd M, McConnell H. Leading Quality Improvement through Best Practice Guideline Development, Implementation, and Measurement Science. Revista MedUNAB. 2015; 17(3):155-162.

19. Cortés OL, Serna A, Salazar LD, Rojas YA, Cabrera S, Arévalo I. Implementación de guías de práctica clínica de la Asociación de Enfermeras de Ontario-RNAO: Una experiencia de enfermería en un hospital colombiano. Revista MedUNAB. 2016; 19(2):103-114.

20. Esparza M, Granados LM, Joya K. Implementación de la guía de buenas prácticas: valoración del riesgo y prevención de úlceras por presión: experiencia en la Fundación Oftalmológica de Santander (FOSCAL). Revista MedUNAB. 2016; 19(2):115-123.

21. Silva A. Formación por competencias en Enfermería. Experiencia de la Universidad de Chile. Revista MedUNAB. 2016;19(2):134-141.

22. Wilson R, Bajnok I, Costa T. Promoting Evidence-Based Care Through Nursing Order Sets. Revista MedUNAB. 2015; 17(3):176-181.

23. Grinspun D. Liderando Cuidados de Enfermería basados en evidencia a través de procesos sistematizados. Revista MedUNAB. 2016; 19(2):83-84.

24. Albornos L, González ME, Moreno T. Implantación de guías de buenas prácticas en España. Programa de centros comprometidos con la excelencia de cuidados. Revista MedUNAB. 2015; 17(3):163-169.

25. Grinspun D, Lloyd M, Xiao S, Bajnok I. Measuring the Quality of Evidence-Based Nursing Care: NQuIRE Nursing Quality Indicators for Reporting and Evaluation Data-System. Revista MedUNAB. 2015; 17(3):170-175.

26. Huky G. Ranking de los sistemas sanitarios más y menos eficientes. [Internet]. 2016. [citado enero de 2017]. Disponible en: https://www.gurusblog.com/archives/ ranking-de-los-sistemas-sanitarios-mas-y-menoseficientes/05/10/2016/ 